\title{
The Progress of Tea Polysaccharide Effect on Anti-aging of Antioxidant
}

\author{
Min Shi , Zenghui Zhao \\ Xi'an Peihua University, Shaanxi Xi'an,china \\ 1243838277@qq.com
}

\begin{abstract}
Key words: Tea PolySaccharide (TPS); Free radicals; Anti-aging; Research progress
\end{abstract}
\begin{abstract}
Tea polysaccharide (TPS), which is considered to be another important active ingredient after Tea Polyphenols Relay, consists of monosaccharides which have a variety of biological activity and the complex structure. It has hypoglycemic, lipid-lowering, anti-thrombosis, anti-oxidation, anti-aging, anti-atherosclerotic, anti-cancer and improve immune function. Modern medical research shows that aging, inflammation, cardiovascular disease, cancer are closely related to the increasing of reactive oxygen species. A large number of natural products in the antioxidant components can clear free radicals, impede the lipid peroxidation by which it was induced, play a preventive role in the occurrence of these diseases. So we review the related literature of TPS in the anti-aging of antioxidants, and aim to provide more reference for the future TPS medicinal research.
\end{abstract}

\section{Introduction}

Aging, that is, aging, is a ubiquitous and irreversible degenerative change in organisms. The aging process is the result of persistent accumulation of degenerative changes in the body, which ultimately leads to decreased organ function and increased susceptibility to disease. With the aging of the body, the adaptability and immunity of the body continue to decline, and the incidence of related degenerative diseases such as cardiovascular diseases, diabetes, Alzheimer's disease, and cancer continues to rise. It ont only seriously affects the quality of life of the elderly, but also significantly increases the prevalence and mortality of the elderly. Aging has become the biggest danger to life. In recent years, research and reports on anti-aging have been increasing. Studies show that aging-related diseases will multiply in the next 20 years[1]. As China's aging population accelerates and social pension pressures increase, how to delay aging, achieve a healthy aging population, and focus on improving the quality of life has become a hot topic in the medical field in recent years.

The current society has emerged a large number of health products for anti-aging, but its components usually contain hormones and have certain toxic and side effects on the body. Therefore, it is particularly necessary to find a safe, reliable and non-toxic natural product to delay aging. Studies have confirmed that natural products mostly reduce oxidative damage through free radical scavenging and thus extend the lifespan of species. A large number of reports have reported that polysaccharides are widely found in animals and plants. Polysaccharides are essential natural macromolecules that constitute living organisms. They can not only maintain many biological functions of organisms, but also have unique biological activities such as anti-oxidation and anti-virus, anti-aging, immune regulation and anti-cancer[2-3]. Tea, one of the three major drinks in the world, is a traditional drink in China. Studies show that tea is rich in tea polyphenols, tea polysaccharides (TPS), amino acids, caffeine and other substances. The TPS is a recognized natural antioxidant and has functions of protecting the liver, reducing blood pressure, reducing blood sugar, 
reducing fat, resisting radiation, preventing canceration, and resisting aging. The anti-aging activity of TPS has been gradually recognized by people. In this article, We will review the recent studies on the anti-aging and anti-aging of TPS, thus providing a basis for delaying the prevention of aging and related diseases.

TPS in tea is considered to be another important active ingredient after tea polyphenols, and study on TPS has attracted great attention. TPS is composed of monosaccharides with various biological activities and complex structures. These monosaccharides are mainly arabinose, galactose and glucose, which have hypoglycemic, lipid-lowering, lipid-lowering, anti-thrombotic, antioxidant, anti-aging, anti-atherosclerosis, anti-cancer and improve immune function. Modern medical research shows that the occurrence of aging, inflammation, cardiovascular diseases, and cancer is closely related to the increase of reactive oxygen free radicals, and antioxidants in a large number of natural products can scavenge free radicals and block the lipid peroxidation triggered by them, to prevent the occurrence of these diseases. It was reported that the antioxidant effect of Green Tea is mainly from tea polyphenols, while the antioxidant effect of Black Tea is mainly from TPS. However, current literature reports on anti-oxidation and anti-aging are mainly concentrated on tea polyphenols, and very few are related to TPS. Therefore, in this paper, we review the research on anti-oxidation and anti-aging of TPS.

\section{Direct removal of reactive oxygen species}

Under normal circumstances, the body has its own antioxidant defense system, which can maintain the body's free radical balance by removing reactive oxygen species (ROS). The antioxidant defense system consists of two antioxidants, one is various enzymes, such as superoxide dismutase (SOD), catalase (CAT) and glutathione peroxidase (GSH-PX), etc; the other is non-enzymes, such as vitamin $\mathrm{C}$, vitamin $\mathrm{E}$, glutathione, uric acid, bilirubin and so on. Among them, SOD is recognized as the primary substance for scavenging free radicals. This enzyme can decompose free radicals to achieve antioxidant functions, and is thus regarded as the "scavengers" of the body. A large number of literature studies have shown that SOD activity is positively correlated with human lifespan and immune function. Different organisms have different SOD activities, and longer-lived people have higher levels of SOD in their liver. For example, mice have low SOD activity, are susceptible to cancer and diseases, and have only a few years of life. In contrast, primates have high SOD activity and longevity up to 50-60 years old; human SOD activity is higher, so life expectancy should be longer. However, with the increase of age, free radicals are generated in the human body and the clearance is reduced. In addition, the activity of SOD in the body continues to decline, resulting in continuous accumulation of ROS and free radicals in the body. The accumulated free radicals damage macromolecules such as cell membranes, intracellular nucleic acids and proteins, triggering lipid peroxidation reactions, and their metabolites affect the expression of DNA and the activity of enzymes, and cause mitochondrial degeneration, rupture, and leakage, thereby interfering with the normal metabolism of cells promotes aging. Therefore, the level of SOD in organisms is usually used as a direct indicator of the aging and death of tissue cells.

Free radical theory believes that excessive production of reactive oxygen in the body or removal too slow will accelerate human aging and associated diseases. Studies showed that TPS is a very good natural antioxidant, and its mechanism of antioxidant activity is related to some of the active sites in TPS (such as amino acids with phenolic hydroxyl groups), which may have captured the free radical. The radicals either generate redox reactions, or TPS macromolecules entrap free radicals, making them unable to undergo oxidation reactions, but the specific mechanism of action is not yet clear. Many scholars have carried out TPS antioxidant experiments in animals and humans. 
The evaluation indexes of TPS antioxidant studies mainly include reactive oxygen species, 1,1-diphenyl-2-trinitrophenylhydrazine (DPPH), and reduction force. The results showed that TPS can significantly remove hydroxyl radical $\left(\mathrm{OH}^{-}\right)$, superoxide anion $\left(\mathrm{O}_{2}{ }^{-}\right), \mathrm{NO}_{2}^{-}, \mathrm{DPPH}$ and other free radicals. Wang et al found that the clearance rate of DPPH free radicals by crude TPS in the concentration range of 25 to $400 \mu \mathrm{g} / \mathrm{ml}$ was $36.58 \%$ to $86.88 \%$, and the scavenging capacity was concentration-dependent[4]. He Xiaomei et al on the in vitro antioxidant activity studies show that TPS has a strong reduction capacity and the ability to scavenge hydroxyl radicals, and with the increase of TPS concentration, its reducing power correspondingly enhanced[5]. When the concentration is $120-160 \mu \mathrm{g} / \mathrm{ml}$, the reducing power of TPS is equivalent to $50 \mu \mathrm{g} / \mathrm{ml}$ vitamin C, and it also has the scavenging effect on nitroso. In vitro studies of Yu Shuchi et al showed that TPS can inhibit the action of strong oxidant $\mathrm{H}_{2} \mathrm{O}_{2}$. With the increase of TPS concentration in Kuding Tea, the inhibitory effect on $\mathrm{H}_{2} \mathrm{O}_{2}$ is gradually enhanced. When the concentration of TPS is $600 \mu \mathrm{g} / \mathrm{ml}$, the antioxidant activity is similar to that of vitamin $\mathrm{C}[6]$. Our previous research also showed that the polysaccharides from Fuzhuan tea had a significant anti-aging effect, the mechanism is probably by scavenging free radicals and its metabolites MDA, SOD and GSH-Px activity increase two-way adjusting effects of aging[7].

\section{Inhibition of lipid peroxidation}

The main active oxygen free radicals in the body are $\mathrm{O}_{2}^{-}, \mathrm{OH}^{-}$, and $\mathrm{H}_{2} \mathrm{O}_{2}$, which can cause lipid peroxidation, resulting in aging and diseases such as vascular sclerosis. Studies have shown that TPS can eliminate excess oxygen free radicals in the body, thus blocking the free radical reaction chain. Excessive reactive oxygen species can cause oxidative stress in peripheral blood lipoproteins, especially the oxidation of low density lipoproteins (LDL) to oxidative LDL, which are important pathogenic factors for atherosclerosis. In vitro experiments by Quan Jishu et al showed that TPS can significantly reduce the plasma levels of very low density lipoprotein cholesterol (VLDL-C) and low density lipoproteins cholesterol (LDL-C) lipid peroxides[8]. When more TPS is absorbed in the body, the antioxidant capacity of the body is stronger and the oxidized LDL-C in the plasma is lower. Furthermore, TPS significantly delays the oxidation modification time of LDL-C, delays the initiation of oxidation modification of LDL-C, and inhibits the oxidation modification of LDL-C. This mechanism plays a pivotal role in explaining the pharmacological effects of TPS such as lipid-lowering, hypoglycemic and prevention of cardiovascular and cerebrovascular sclerosis. Compared with the high-fat model and the $\mathrm{CCl}_{4}$-induced liver injury mouse model, TPS has been reduced to varying degrees in the treatment of evaluation indicators such as serum total cholesterol (TC), LDL-C, triglyceride (TG), aspartate aminotransferase, alanine aminotransferase. Furthermore, the expression of SOD, GSH-Px, and CAT in liver and heart tissues increased significantly, whereas hepatic lipid peroxidation products, $15-\mathrm{F}_{2 \mathrm{t}}$-specific prostaglandins, and MDA in liver decreased significantly. Thus improving the body's antioxidant activity and inhibiting the damage of lipid peroxidation to the liver.

\section{Activate the antioxidant defense system}

Antioxidant defense system exists in the cells, which can remove excess free radicals in the body and maintain the dynamic balance of free radicals. In addition to antioxidant defense system including SOD, CAT, GSH-PX, etc., there are some non-enzymatic compounds (vitamin E, vitamin C, etc.), and TPS anti-aging effect is borne by activating the antioxidant enzyme defense system and scavenging free radicals. Sun Hongmei's study shows that TPS has positively improved the 
free-radical metabolism and anaerobic exercise capacity of Chinese wrestling female athletes before the game by enhancing anti-oxidant capacity. In this study, the author selected 20 elite Chinese wrestling female athletes as the study subjects. After 4 weeks of pre-training, the levels of SOD, GSH-Px, CAT, GSH, and glutathione reductase (GR) in plasma of TPS group were significantly higher and the MDA was significantly lower. It was concluded that TPS intervention can promote the synthesis of red blood cells and hemoglobin during the pre-competition training period, thereby reducing skeletal muscle, myocardium and liver damage caused by oxidative stress. Ren et al found that Selenium-enriched Tea TPS can significantly increase the activity of SOD and GSH-Px in diabetic mice liver and kidney, and reduce the lipid peroxidation product malondialdehyde (MDA). This can reduce the damage of oxidative stress to islet tissue in diabetic mice[9].

\section{Regulate body immunity to delay aging}

In the 1960s, Walford proposed the theory of aging immunology, which considered that the immune system is a conditioning device during the aging process and is characterized by ageing changes. The immune system during aging is mainly manifested in two aspects: First, the decline of normal immune function, such as immune organs (thymus, lymph node) atrophy and fibrosis, decreased immune cells, immune response lag, decreased immune function; Second, the autoimmune response is too strong, mainly manifested as increased immunity to autoantigens, but the immune response to foreign antigens declined.

The immune system is an important system for the body to carry out immune responses and realize immune functions. It consists of immune organs, immune cells, and immune molecules. At present, the immunomodulation of polysaccharides of natural products has become a research hotspot in life sciences field. A large number of studies have shown that TPS can not only scavenge free radicals, but also activate immune cells such as T/B lymphocytes, macrophages and natural killer (NK) cells, thereby improving the body's immune function and delaying aging[10]. The immune regulation of polysaccharides is inseparable from its receptors. First, it binds to receptors on the cell surface and activates intracellular signaling pathways. It activates immune cells and promotes the expression of cytokines. In recent years, Toll-like receptors (TLR) have been identified as a very important transmembrane signal transduction receptor that can recognize and bind pathogen-associated molecular patterns (PAMP) and play a pivotal role in innate immunity and adaptive immunity. TLR7 is a target of TPS on macrophages. It activates T/B lymphocytes, macrophages, and NK cells, increases the ratio of $\mathrm{CD}_{4}+/ \mathrm{CD}_{8}+$ lymphocytes, promotes the secretory and expression of IgG, NO, and alpha tumor necrosis factor (TNF- $\alpha$ ), interleukin-6 (IL-6), and NF-E2-associated factor 2 (Nrf2), and plays a regulatory role in the immune system. However, TPS has different expressions of immunomodulatory activity in the body. Tea tree polysaccharides, enzymatically modified polysaccharides and Pyracantha polysaccharides can significantly enhance the body's immune activity.

The occurrence and development of tumors are closely related to the body's immune function. TPS can exert its anti-tumor efficacy by promoting the secretion and expression of immune cytokines. Shen Jian et al used a tumor-bearing mouse model and given TPS intervention, it showed that TPS can not only significantly increase the spleen index and serum IgG content in mice, but also induce splenocytes of mice to produce important cytokines IL-2 and TNF- $\alpha$ [11]. IL-2 can further induce the proliferation of Th cells and Tc cells, thereby stimulating the production of antibodies by B cells, enhancing the activity of NK cells and LAK cells and inducing interferon production and other effects. TNF- $\alpha$ has immunomodulatory and immune effector functions that regulate macrophages and activate the body's immune response. 


\section{Summary and prospect}

With the rapid development of modern medical biotechnology, under the impetus of cells and molecular biology, a breakthrough has been made in the mechanism of aging from a microscopic point of view. Scientists put forward a variety of valuable theories, such as free radical theory, Mitochondrial DNA damage theory, telomere theory, biomembrane damage theory, immunology theory, chromosome mutation theory, genetic programming theory. Among these doctrines, the most representative is free radical theory.

The theory of free radicals was proposed by Harman in 1956. It is the most widely accepted and widely studied theory. The theory is that in the process of oxygen metabolism, most of the oxygen metabolism produces ATP for the body, and about $2 \%$ of oxygen metabolism produces ROS and superoxide radicals $\left(\mathrm{H}_{2} \mathrm{O}_{2}\right.$, hydroxyl radicals). ROS can cause serious damage to biological structures, such as cell membrane damage, DNA degeneration and breakage, chromosome translocation, etc., become a key factor in the toxicity of oxygen accelerated aging. ROS is produced during oxygen metabolism and forms in the mitochondria of cells in the electron transport chain. When the electron transfer efficiency is low, a large amount of superoxide radicals are formed, they further form ROS, and ROS can damage mitochondria. When the mitochondria are damaged, the efficiency of their electron transfer chains is reduced accordingly. Then increase of ROS production and increase of mitochondrial oxidative damage, thereby forming a vicious cycle to promote aging. A large number of studies have shown that the mechanism of aging is most closely related to the accumulation of free radicals in the body, mitochondrial damage, and dysfunction[12].

In summary, TPS can significantly increase antioxidant markers such as SOD, GSH-Px, and CAT, reduce over-oxidation markers such as MDA, TC, and LDL-C, and exhibit strong antioxidant activity. Therefore, TPS has a good anti-aging effect and has broad application prospects. Although there are not enough studies on the anti-aging of TPS, the study on the antioxidant mechanism of TPS proves that TPS has the effect of prolonging life, and provides scientific basis for TPS in anti-aging research. However, the research on anti-aging application of TPS is not perfect enough, especially the TPS intervention studies and clinical randomized controlled trials (RCT) lack of large-sample aging animal model, thus restricting the development and utilization of TPS to some extent. China is the country with the highest tea production in the world, and can produce 650,000 tons of tea each year. TPS is particularly rich in crude old tea, but with the improvement of people's living standards, the using of crude old tea is becoming less and less. The use of crude old tea resources to extract TPS, especially for the full use of middle and low grade tea, is of great significance. TPS is an inexpensive and effective compound. We must carry out in-depth research on the extraction, isolation, and biological activity of TPS, and develop health-promoting foods and drugs with anti-aging effects, making TPS an important part of our healthy life. At the same time, the full use of tea resources not only improves economic efficiency, but also has important significance for disease prevention and promotion of human health.

\section{Acknowledgment}

This work is supported by Shaanxi Provincial Department of Education support project (No. 17JK1057), all support is gratefully acknowledged. 


\section{Reference}

[1] de Cabo R, Carmona-Gutierrez D, Bernier M, et al. The searchfor anti-aging interventions: from elixirs to fasting regimens[J]. Cell, 2014, 157( 7) : 1515-1526.

[2] You Lijun, Gao Qing, Feng Mengying, et al. Structural characterisation of polysaccharides from tricholoma matsutake andtheir antioxidantand antitumour activities[J]. Food Chem, 2013, 138(4):2242-2249.

[3] Wu Wenlin, Zhu Yuanting, Zhang Li, et al. Extraction, preliminary structural characterization, and antioxidant activities of polysaccharides from Salvia miltiorrhiza Bunge[J].Carbohydrate Polymers, 2012, 87(2):1348-1353.

[4] Wang YF, Li YF, Liu YY, et al. Extraction, characterization and antioxidant activities of Se-enriched tea polysaccharides[J]. International Journal of Biological Macromolecules, 2015, 77(6182): 76-84.

[5] He Xiaomei, Zhang Ying, Xu Xingyun, et al. Study on enzyme-assisted extraction and antioxidant activity of low-grade green tea polysaccharides[J]. Food Industry science and technology, 2015, 36(10): 153-157.

[6] Yu Shuchi, Hou Jinxin. Study on scavenging effects of longjing tea polysaccharides on free NO2[J]. Food Research and Development, 2012, 33(4): 28-31.

[7]Shi Min, Chen Lei, Guo Xiaobo, et al. Study on Anti-aging Effect of Jingwei Fuzhuan Tea Polysaccharides in D-galactose induced aging mice model[J]. Chinese Journal of Aesthetic Medicine, 2018, 27(01): 81-84

[8] Quan Jishu, Yin Xuezhe, Jin Zewudao. Study on antioxidant effect of tea polysaccharides[J]. Chinese herbal medicine, 2007, 30(9): 1116-1118.

[9] Ren DY, Hu YY, Luo YY, etal. Selenium-containing polysaccharides from Ziyang green tea ameliorate high-fructose diet induced insulin resistance and hepatic oxidative stress in mice[J]. Food \& Function, 2015, 6(10): 3342-3350.

[10] Yu Dongning, Chen Ping, Wang Shuang, et al. Effect of Longjing Tea Polysaccharide on Oxidative Stress in ALX Diabetic Mice [J]. Chinese Journal of Food Science, 2016, 16(4): 30-34.

[11] Shen Jian, Chen Zengliang, Shen Xiangdi, et al. Tea polysaccharide antitumor and its enhancing effect on immunity[J]. Zhejiang Preventive Medicine, 2007, 19(8): 10-12.

[12] Gustavo B. The mitochondrial free radical theory of aging[J]. ProgMol Biol Transl Sci, 2014, (127) : 1-27. 